\title{
Evaluating the accuracy of three international guidelines in identifying the risk of malignancy in pancreatic cysts: a retrospective analysis of a surgical treated population
}

\author{
A.Vanden Bulcke1, J. Jaekers ${ }^{2}$, H. Topal ${ }^{2}$, D. Vanbeckevoort ${ }^{3}$, V. Vandecaveye ${ }^{3}$, T. Roskams ${ }^{4}$, B.A. Weynand ${ }^{4}$, J. Dekervel ${ }^{1}$, \\ E. Van Cutsem ${ }^{1}$, H. van Malenstein ${ }^{1}$, C. Verslype ${ }^{1}$, W. Laleman ${ }^{1}$, S. van der Merwe \\ (1) Department of gastroenterology and hepatology, UZ Leuven, Herestraat 49, 3000, Leuven, Belgium ; (2) Department of hepatobiliary and pancreatic surgery, UZ \\ Leuven, Herestraat 49, 3000, Leuven, Belgium ; (3) Department of radiology, UZ Leuven, Herestraat 49, 3000, Leuven, Belgium ; (4) Department of pathology, UZ Leuven, \\ Herestraat 49, 3000, Leuven, Belgium.
}

\begin{abstract}
Background and study aims: The international consensus Fukuoka guideline (Fukuoka ICG), The European evidence-based guideline on pancreatic cystic neoplasms (European EBG) and the American Gastroenterological Association institute guideline on the diagnosis and management of asymptomatic neoplastic pancreatic cysts (AGA IG) are 3 frequently cited guidelines for the risk stratification of neoplastic pancreatic cysts. The aim of this study was to assess the accuracy of detecting malignant cysts by strictly applying these guidelines retrospectively to a cohort of surgically resected pancreatic cysts.

Patient and methods: 72 resected cysts were included in the analysis. Invasive carcinoma, high grade dysplasia and neuro-endocrine tumour were considered as "malignant cysts" for the purpose of the study.

Results: $32 \%$ of the resected cysts were malignant. The analysis showed that the Fukuoka ICG, European EBG and AGA IG had a sensitivity of $66,8 \%, 95,5 \%, 80 \%$; a specificity of $26,8 \%, 11,3 \%$, $43,8 \%$; a positive predictive value of $31,8 \%, 35 \%, 47,1 \%$ and a negative predicted value of $61,1 \%, 83,3 \%, 77,8 \%$ respectively. The missed malignancy rate was respectively $11,3 \%, 1,5 \%, 7,7 \%$ and surgical overtreatment was respectively 48,4\%, 59,1\%, 34,6\%.

Conclusion: In this retrospective analysis, the European EBG had the lowest rate of missed malignancy at the expense of a high number of "unnecessary" resections. The Fukuoka ICG had the highest number of missed malignancy. The AGA IG showed the lowest rate of unnecessary surgery at the cost of a high number of missed malignancy. There is need to develop better biomarkers to predict the risk of malignancy. (Acta gastroenterol. belg., 2021, 84, 443-450).
\end{abstract}

Key Words: IPMN, malignancy risk, surgery, overtreatment.

\section{Introduction}

The challenge in the management of pancreatic cysts remains to determine which cysts are at risk of developing invasive cancer and how surveillance can be optimized. The purpose of this policy is to prevent pancreatic cancer by timely surgery and minimise the morbidity and mortality associated with unnecessary surgery. This is not an easy undertaking as it requires an accurate diagnosis of the type of cyst as well as the establishment of the underlying predisposition for a particular cyst to progress to malignancy over time. In clinical practice this mostly refers to intraductal papillary mucinous neoplasm (IPMN) - subdivided in main duct IPMN (MD-IPMN), branch duct IPMN (BD-IPMN) and mixed-type IPMN - mucinous cystic neoplasm (MCN), cystic pancreatic neuroendocrine tumour (NET) and cystic degeneration of a pancreatic adenocarcinoma. These cystic lesions need to be differentiated from benign cystic lesions such as serous cystic neoplasm ( $\mathrm{SCN}$ ) having no malignant potential. SCN needs no follow up and a resection is only required if symptomatic $(1,2)$. Given that the annual progression rate of a BD-IPMN towards high grade (HG) dysplasia or adenocarcinoma is low (around 1,4-6,9\%), it is evident that inappropriate surgical resection will be associated with increased morbidity and mortality (3). On the other hand unduly delayed surgery, when a cyst is progressing to malignancy, will prevent the opportunity for curative surgery. It is for this reason that the major societies in pancreatology have devised guidelines to aid physicians in correctly assigning patients to either surveillance or resection care paths.

The three most cited guidelines for risk stratification and management of neoplastic cysts are the International consensus Fukuoka guidelines from the International Association of Pancreatology (Fukuoka ICG) updated in 2017, the European evidence-based guidelines on pancreatic cystic neoplasms from The European Study Group on Cystic Tumours of the Pancreas (European EBG) updated in 2018 and the American gastroenterological association institute guideline on the diagnosis and management of asymptomatic neoplastic pancreatic cysts (AGA IG) - published in 2015 (3-5).

The risk stratification of mucinous cysts (IPMN and $\mathrm{MCN}$ ) is not straightforward and the three guidelines differ in this respect. The sensitivity in detecting $\mathrm{HG}$ dysplasia and malignant cysts is overall good, but at the expense of a low specificity resulting in a high number of unnecessary resections $(3,6,7)$.

The aim of this study was to assess the accuracy of the three most common guidelines in identifying $\mathrm{HG}$ dysplasia and malignant cysts retrospectively in a cohort of resected pancreatic cysts.

Correspondence to : Schalk van der Merwe, Department of gastroenterology and hepatology, Herestraat 49, 3000 Leuven, Belgium. Phone : +32 (0)16 344775. Fax : +32 (0)16 344419 .

E-mail: schalk.vandermerwe@uzleuven.be

Submission date : 05/10/2020

Acceptance date : 02/01/2021 


\section{Subjects and methods}

This retrospective study was approved by the local Ethics Committee Research on 14/02/2017.

\section{Patient selection}

A retrospective analysis was performed on a population of surgical resected pancreatic cysts from august 2011 until august 2016. All cysts were resected in an expert academic reference centre for pancreatic surgery. In this centre more than 100 pancreatic resections are performed annually. Cysts were considered for inclusion in the analysis if a preoperative diagnostic workup was performed in order to identify the aetiology and to estimate the malignancy risk of the cyst. Symptomatic and asymptomatic cysts were both included. Cysts were excluded from analysis if the preoperative examinations were not available for review and if there was no clear visible cystic lesion on preoperative imaging.

All pancreatic tissue specimens received by the department of pathology during this period were reviewed to compose the study population. Of the 755 available reports of pancreatic samples, a total of 76 resected pancreatic cysts were identified of whom 72 were eligible for analysis (figure 1). Information was retrieved from the institutional electronic patient files to construct the database. In the event of discrepancy the case was reviewed by the senior author.

\section{Definitions}

Symptomatic cyst: Symptoms that may be associated with a pancreatic cyst are weight loss, pancreatic like

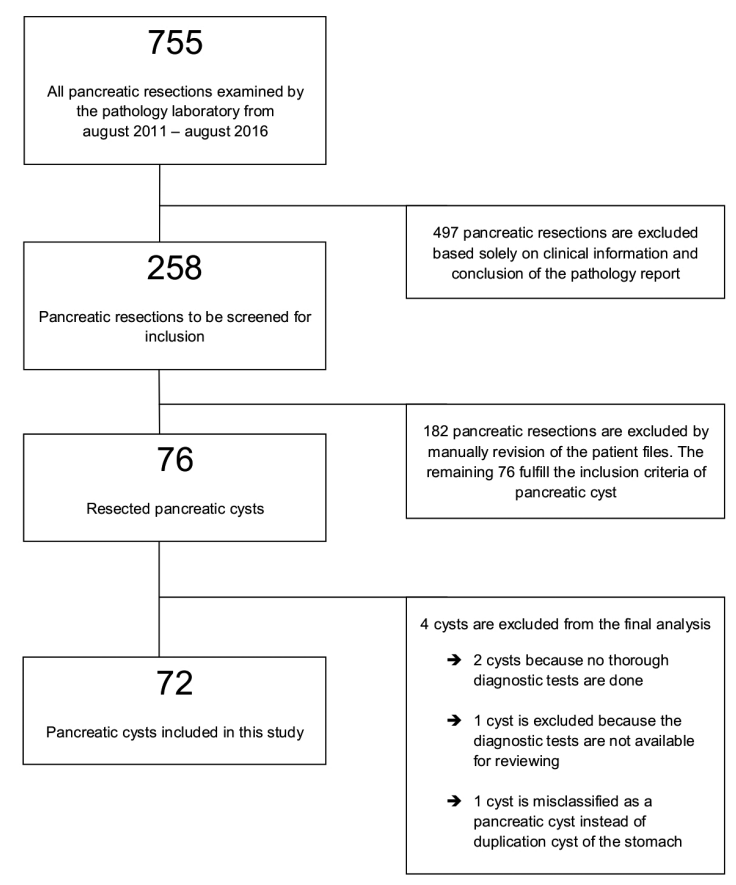

Figure 1. - Schematic presentation of how the study population was composed. pain, pancreatitis, icterus, steatorrhea and new onset or fast deterioration of diabetes mellitus. 'Pancreatic like pain' is defined as a gnawing pain in the upper abdomen, radiating to the back and often with a relation to meal intake.

Size of the cyst: The largest diameter of the cyst measured on magnetic resonance imaging (MRI), computed tomography (CT) or endoscopic ultrasound (EUS). For the MD-IPMN, only the width of the main pancreatic duct (MPD) is taken into account and not the length of the main duct involvement.

Location: The proximal pancreas is defined as the part of the pancreas situated at the right side of the superior mesenteric artery and consists of the uncinate process, the head and the neck of the pancreas. The distal pancreas consists of the part of the pancreas situated at the left side of the superior mesenteric artery, consisting of the body and tail. In case a pancreas is diffusely involved with IPMN, the location of the cyst is classified as total pancreatic involvement.

Dilatation and relation to the main pancreatic duct: Primary dilation is defined as a focal dilatation of the MPD without clear obstruction downstream and without dilatation upstream of the cyst. Secondary dilatation is defined as dilation of the MPD upstream of a cystic lesion or stenosis. A combination of primary and secondary dilatation is possible and is referred to "combined dilatation".

Malignant cysts: cysts consisting of adenocarcinoma, HG dysplasia or NET. Premalignant cysts: This group consists of MCN or IPMN with low grade (LG) dysplasia. Benign cysts: cysts with no malignant potential (e.g. $\mathrm{SCN}$, pancreatic pseudocyst, retention cyst etc).

Avoidable resection: A resected non-malignant cyst with non-fulfilled criteria of the involved guideline.

Correct referral: A resected malignant cyst with fulfilled criteria of the involved guideline.

Overtreatment: A resected non-malignant cyst with fulfilled criteria of the involved guideline.

Missed malignancy: A resected malignant cyst with non-fulfilled criteria of the involved guideline.

\section{Application of the criteria}

The three guidelines estimate the risk of malignancy by high risk stigmata and worrisome features. The interpretation and decisiveness of each feature can differ between the guidelines and can lead to different treatment advice for the same cyst. In our analysis, we adopted the flowchart of each guideline strictly to decide if there was a proper indication for referral to surgery.

A lesion can only be analysed following a guideline if all the necessary diagnostic information is available to complete the flowchart. Table 1 summarizes the general principles of the three guidelines for mucinous cysts. We highlight the following differences between the guidelines: 
Table 1. - Summary of the guidelines for mucinous cysts

\begin{tabular}{|c|c|c|c|}
\hline Guideline & Type of cyst & $\begin{array}{c}\text { Absolute indication for surgery } \\
\text { High risk factors }\end{array}$ & $\begin{array}{c}\text { Relative indication for surgery } \\
\text { worrisome features }\end{array}$ \\
\hline Fukuoka ICG 2017 (3) & IPMN & $\begin{array}{l}\text { Jaundice, } \mathrm{MPD} \geq 10 \mathrm{~mm} \text {, Nodule } \geq 5 \mathrm{~mm} \\
\Rightarrow \quad \begin{array}{l}\text { Refer to surgery if } 1 \text { High risk factor is } \\
\text { positive }\end{array}\end{array}$ & $\begin{array}{l}\text { Cyst } \geq 30 \mathrm{~mm}, \text { nodule }<5 \mathrm{~mm} \text {, thickened cystic } \\
\text { walls, MPD 5-9,9 mm, lymphadenopathy, } \\
\text { pancreatitis, elevated CA 19.9, cyst growth } \geq 5 \\
\text { mm/2 years } \\
\Rightarrow \text { If } 1 \text { worrisome feature is positive, } \\
\quad \text { confirm by EUS } \\
\Rightarrow \text { If EUS confirms worrisome features, } \\
\text { refer to surgery }\end{array}$ \\
\hline European EBG 2018 (4) & IPMN & $\begin{array}{l}\text { Jaundice, MPD } \geq 10 \mathrm{~mm} \text {, Nodule } \geq 5 \mathrm{~mm} \text {, solid } \\
\text { mass, positive cytology (HG dysplasia or invasive } \\
\text { malignancy) } \\
\Rightarrow \quad \text { Absolute indication for surgery if } 1 \mathrm{High} \\
\text { risk factor is positive }\end{array}$ & $\begin{array}{l}\text { Cyst } \geq 40 \mathrm{~mm} \text {, Nodule }<5 \mathrm{~mm} \text {, MPD } 5-9,9 \\
\mathrm{~mm} \text {, elevated CA } 19.9 \text {, pancreatitis, new onset } \\
\text { Diabetes mellitus, cyst growth } \geq 5 \mathrm{~mm} / 1 \text { year } \\
\Rightarrow \text { If } 1 \text { worrisome feature is positive, } \\
\text { relative indication for surgery in } \\
\text { patient with no comorbidities }\end{array}$ \\
\hline AGA IG 2015 (5) & IPMN & $\begin{array}{l}\text { All MD-IPMN } \\
\text { BD-IPMN: Cyst } \geq 30 \mathrm{~mm} \text {, dilatation MPD, solid } \\
\text { mass } \\
\Rightarrow \quad \text { If } 2 \text { High risk factors are positive and } \\
\\
\text { concerning features are confirmed by } \\
\quad \text { EUS } \\
\quad \text { (No confirmation by EUS needed for } \\
\text { solid mass together with a dilated MPD) }\end{array}$ & \\
\hline Fukuoka ICG 2017 (3) & $\mathrm{MCN}$ & Refer all to surgery & \\
\hline European EBG 2018 (4) & $\mathrm{MCN}$ & $\begin{array}{l}\text { Cyst } \geq 40 \mathrm{~mm} \text {, mural nodule, symptomatic } \\
\quad \Rightarrow \quad \text { Refer to surgery if } 1 \text { high risk factor is } \\
\text { positive }\end{array}$ & \\
\hline AGA IG 2015 (5) & $\mathrm{MCN}$ & No strategy mentioned & \\
\hline
\end{tabular}

IPMN : Intraductal Papillary Mucinous Neoplasm, MCN : Mucinous Cystic Neoplasm, MPD : Main Pancreatic Duct, MD-IPMN : Main Duct IPMN, BD-IPMN : Branch Duct IPMN, EUS : Endoscopic ultrasound, CA 19.9 : Carbohydrate antigen 19.9.

For the Fukuoka ICG guidelines: all MCN, regardless of size or presence of worrisome features justify surgery. In case of a BD-IPMN with worrisome features, an EUS +/- fine needle aspiration (FNA) is necessary before the guideline can be applied. The purpose of this EUS is to confirm the worrisome aspect of the cyst. This includes confirmation of a mural nodule $\geq 5 \mathrm{~mm}$ (and differentiating it from mucin plugs), obtaining of cytology at least suspicious for malignancy or demonstrating main duct involvement by showing a thickened wall, intraductal mucin or mural nodules. If the result of the EUS is negative, the Fukuoka ICG does not advice surgery. If the EUS is inconclusive or the cyst is larger than 30 $\mathrm{mm}$ without confirmation by EUS of worrisome features, the guideline gives the choice between surveillance and consideration for surgery. In the setting of our study, such cysts were eligible for follow up and were not advised to undergo surgery (3).

For the European EBG: although this guideline is comparable to the Fukuoka ICG, some differences need to be highlighted. An asymptomatic $\mathrm{MCN}$ smaller than 40 $\mathrm{mm}$, without a mural nodule can be followed up (instead of referring all MCN's to surgery in the Fukuoka ICG). For BD-IPMN, a diameter of $\geq 40 \mathrm{~mm}$ is considered as worrisome (instead of $\geq 30 \mathrm{~mm}$ in the Fukuoka ICG). A MD-IPMN is considered worrisome if the MPD is dilated to more than $5 \mathrm{~mm}$. Another important difference between the European EBG and the Fukuoka ICG is that one worrisome feature on CT scan or MRI scan in a patient without comorbidities is sufficient for referring to surgery without the need of confirmation by EUS (4).

The AGA IG, compared to the other two guidelines, is simplified regarding detailed description of individual criteria and the guideline does not mention a strategy for MCN. Hence, MCN are excluded in our analysis of the AGA IG. MD-IPMN is an indication for surgery. For BD-IPMN, the guideline does not differentiate between high risk and worrisome features but considers cysts with two high risk factors (cyst $\geq 30 \mathrm{~mm}$, dilatation of the MPD and solid mass/nodule) confirmed by EUS/ FNA as an indication for surgery. A solid mass/nodule in combination with a dilated MPD does not need EUS/FNA confirmation and can be referred directly for surgery. If only one high risk factor is present, the guideline advises no referral to surgery (5). The guideline does not mention a cut off diameter for the dilatation of the MPD. We used in our analysis a cut off of $5 \mathrm{~mm}$. To note that the AGA guideline concerns only asymptomatic cysts. For this analysis, we applied the AGA IG guidelines also on symptomatic cysts.

\section{Statistical analysis}

The performance of the guidelines was assessed by calculating the sensitivity, specificity, positive predictive value (PPV), negative predictive value (NPV) and accuracy. The accuracy is the proportion of the sum of true positives and true negatives in comparison with the whole test group. All graphs were designed with Microsoft ${ }^{\circledR}$ Excel ${ }^{\circledR} 2007$. 
Table 2. - Patient and cyst characteristics

\begin{tabular}{|c|c|c|c|}
\hline Total cysts & $\mathbf{N}$ & 72 & \\
\hline \multicolumn{4}{|l|}{ PATIENT CHARACTERISTICS } \\
\hline Mean age & Years & 61 & $7-81(\mathrm{SD}=13,6)$ \\
\hline \multicolumn{4}{|l|}{ Sex } \\
\hline Female & $\%(\mathrm{~N})$ & $48,7 \% \quad(35)$ & \\
\hline Male & $\%(\mathrm{~N})$ & $51,2 \% \quad(37)$ & \\
\hline $\begin{array}{l}\text { Incidentally diagnosed } \\
\text { Diagnosis because of symptoms }\end{array}$ & $\begin{array}{l}\%(\mathrm{~N}) \\
\%(\mathrm{~N})\end{array}$ & $\begin{array}{ll}50 \% & (36) \\
50 \% & (36)\end{array}$ & \\
\hline Unclear symptom association & $\%(\mathrm{~N})$ & $9,7 \% \quad(7)$ & \\
\hline Likely associated symptoms & $\%(\mathrm{~N})$ & $40,3 \% \quad(29)$ & \\
\hline Weight loss & $\%(\mathrm{~N})$ & $15,2 \% \quad(11)$ & \\
\hline Pancreatitis & $\%(\mathrm{~N})$ & $25 \% \quad(18)$ & \\
\hline Pancreas like pain & $\%(\mathrm{~N})$ & $19,4 \% \quad(14)$ & \\
\hline Icterus & $\%(\mathrm{~N})$ & $5,6 \% \quad(4)$ & \\
\hline Steatorrhea & $\%(\mathrm{~N})$ & $2,8 \% \quad(2)$ & \\
\hline Rapid evolving diabetes & $\%(\mathrm{~N})$ & $4,2 \%$ & \\
\hline $\begin{array}{l}\text { Referral } \\
\text { In hospital referral } \\
\text { Extern referral }\end{array}$ & $\begin{array}{l}\%(\mathrm{~N}) \\
\%(\mathrm{~N})\end{array}$ & $\begin{array}{ll}26 \% & (19) \\
74 \% & (53) \\
\end{array}$ & \\
\hline $\begin{array}{l}\text { Surgery at the time of diagnosis } \\
\text { Surgery after surveillance program }\end{array}$ & $\begin{array}{l}\%(\mathrm{~N}) \\
\%(\mathrm{~N})\end{array}$ & $\begin{array}{ll}75 \% & (54) \\
25 \% & (18)\end{array}$ & \\
\hline Mean duration of follow up & months & 27,9 & $3-91(\mathrm{SD}=24,7)$ \\
\hline \multicolumn{4}{|l|}{ CYST CHARACTERISTICS } \\
\hline Mean cyst size & $\mathrm{mm}$ & 39,6 & $17-170(\mathrm{SD}=27,9$ \\
\hline Main duct dilatation & $\%(\mathrm{~N})$ & $56,9 \%(41)$ & \\
\hline Primary dilatation & $\%\left(\mathrm{~N}^{*} / \mathrm{N}\right)$ & $17 \%(7)$ & \\
\hline Secondary dilatation & $\%\left(\mathrm{~N}^{*} / \mathrm{N}\right)$ & $34 \%(14)$ & \\
\hline Combined dilatation & $\%(\mathrm{~N} * / \mathrm{N})$ & $49 \%(20)$ & \\
\hline Mean diameter main duct & $\mathrm{mm}$ & 7,8 & $3-20(\mathrm{SD}=3,66)$ \\
\hline \multicolumn{4}{|l|}{ Location of the cyst - type of surgery } \\
\hline Proximal pancreas & $\%(N)$ & $50 \%(36)$ & \\
\hline Whipple resection & $\%(\mathrm{~N})$ & $44 \%(32)$ & \\
\hline Total pancreatectomy & $\%(\mathrm{~N})$ & $3 \% \quad(2)$ & \\
\hline Enucleation & $\%(\mathrm{~N})$ & $3 \% \quad(2)$ & \\
\hline Distal Pancreas & $\%(\mathrm{~N})$ & $46 \%(33)$ & \\
\hline Tail resection & $\%(\mathrm{~N})$ & $37 \%(27)$ & \\
\hline Central pancreatectomy & $\%(\mathrm{~N})$ & $6 \%(4)$ & \\
\hline Enucleation & $\%(\mathrm{~N})$ & $3 \%(2)$ & \\
\hline Total Pancreas & $\%(\mathrm{~N})$ & $4 \%(3)$ & \\
\hline Total pancreatectomy & $\%(\mathrm{~N})$ & $4 \%(3)$ & \\
\hline
\end{tabular}

$\mathrm{N}$ : number of cysts, SD : Standard Deviation

\section{Results}

Preoperative investigation: During the preoperative evaluation, a CT scan was performed in 73,6\% (53/72), a MRI scan in $88,9 \%(64 / 72)$, EUS in $75 \%(54 / 72)$, FNA in $45,8 \%(33 / 72)$ and the indication for surgery was discussed on our multidisciplinary tumour board in $58,3 \%(42 / 72)$ of the cases. 14 of the 33 cytology samples $(42 \%)$ derived from FNA were considered as "not adequate enough for assessment" due to a low number or absence of cells.

Patient characteristics: For the details, we refer to table 2. To highlight that $50 \%(36 / 72)$ of the cysts were symptomatic. For $1 / 5^{\text {th }}$ of these cases, the association of the reported symptoms were in retrospect unclear. $25 \%$ $(18 / 72)$ of the cysts were referred from a surveillance program.
Cyst characteristics: For the details, we refer to table 2.

Pathology outcome: The majority of the resected cysts were mucinous: $71 \%$ (51/72) were IPMN, 12\% (9/72) MCN. The other cysts comprised 2 SCN, 2 NET, 3 chronic pancreatitis, 4 benign epithelial/congenital cysts and 1 pseudocyst (table 3 and figure 2). 14\% (10/72) of the resected cysts were benign, 54\% (39/72) were premalignant and $32 \%(23 / 72)$ were malignant cysts (figure 3 ). The malignant subgroup consisted of 11 adenocarcinomas, $10 \mathrm{HG}$ dysplasias and 2 NETs. If the NETs are ignored, the 21 remaining malignant cysts were all IPMN of which the majority had main duct involvement: 9 were MD-IPMN, 8 were mixed-type IPMN and 4 malignant cysts originated from BD-IPMN (figure 3 ). Out of the 18 cysts referred from a surveillance program, 3 were benign (a SCN, a bleeding cyst and a 
Table 3. - Final pathological diagnosis (see also figure 2)

\begin{tabular}{|l|c|c|}
\hline Neoplastic cysts & $51(71 \%)$ & \\
\hline IPMN & & $43 \%(22 / 51)$ \\
BD-IPMN & & $24 \%(12 / 51)$ \\
MD-IPMN & $9(12 \%)$ & $33 \%(17 / 51)$ \\
Mixed-type IPMN & $2(3 \%)$ & \\
MCN & $2(3 \%)$ & \\
SCN (*) & \\
NET & $3(4 \%)$ & \\
\hline Non-Neoplastic cysts & $4(6 \%)$ & \\
\hline Chronic Pancreatitis & \\
Benign epithelial/congenital cyst & $1(1 \%)$ & \\
pseudocyst (=bleeding cyst) & 72 & \\
\hline Total &
\end{tabular}

(*) SCN is considered as a benign lesion. IPMN : Intraductal papillary mucinous neoplasm, MCN : Mucinous cystic neoplasm, MD-IPMN : Main duct IPMN, BD-IPMN : Branch duct IPMN, SCN : Serous cystic neoplasm, NET : Neuroendocrine tumour.

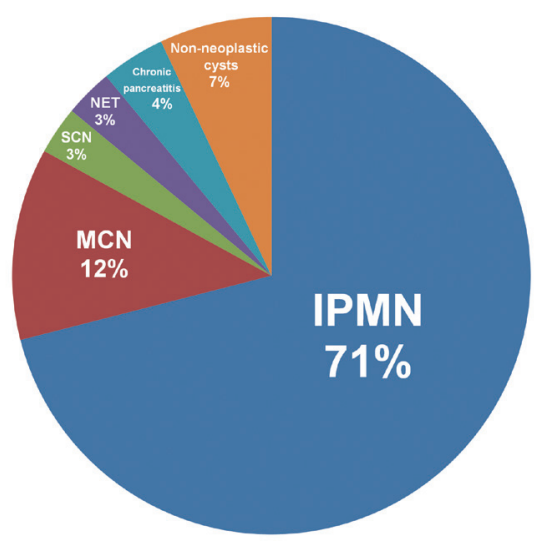

Figure 2. - Final pathological diagnosis : 72 resected pancreatic cysts are included in this analysis. The pathological etiology of the cysts is proportionally shown in the figure (see also table 3). IPMN : Intraductal papillary mucinous neoplasm, MCN : Mucinous cystic neoplasm, SCN: Serous cystic neoplasm, NET : Neuroendocrine tumour.

chronic pancreatitis), 11 were premalignant cysts and only 4 were malignant. If we analyse the group of cysts with dilated main pancreatic duct (total of 41/72 cysts),
A

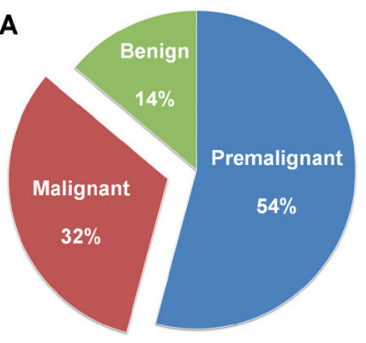

B
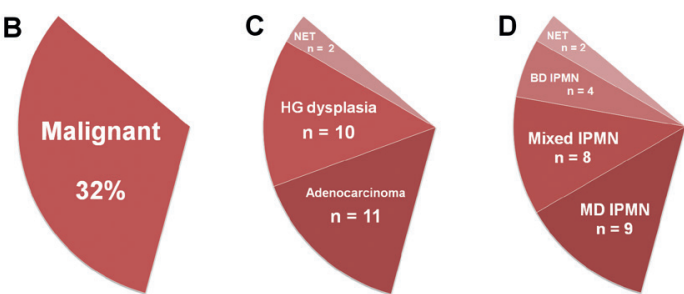

Figure 3. - Characteristics of the malignant cysts : A + B : 23 resected pancreatic cysts $(=32 \%)$ were malignant on a total of 72 pancreatic cysts. 39 cysts $(=54 \%)$ were premalignant and 10 cysts $(=14 \%)$ were benign. $\mathrm{C}: 11$ of the 23 resected malignant cysts were adenocarcinoma, 10 were high grade dysplasia and 2 were NET. D : 17 of the resected malignant mucinous cysts had main duct dilatation of whom 9 were MD-IPMN and 8 mixed IPMN. 4 resected malignant mucinous cysts were BD-IPMN. IPMN : Intraductal papillary mucinous neoplasm, MD-IPMN : main duct IPMN, BD-IPMN : branch duct IPMN, NET : Neuroendocrine tumour, HG dysplasia : High grade dysplasia.

19 cysts were malignant and 22 cysts non-malignant. If we compare the width of the dilatation in both groups, there is no clinical important difference: the dilatation of the malignant subgroup is 7,69 $\mathrm{mm}$ (Standard deviation (SD) $3,77 \mathrm{~mm}$ ) compared to a dilatation of 7,78 $\mathrm{mm}$ (SD $3,70)$ of the non-malignant group.

Prediction of malignancy: Regarding the primary research question of this analysis, we retrospectively applied the criteria of the three guidelines to evaluate the accuracy of predicting malignancy. Results are shown in table 4.

Table 4. - Predictability of the three guidelines

\begin{tabular}{|l|c|c|c|c|c|c|c|}
\hline & $\mathrm{N}$ & Criteria + & Malignancy & Sensitivity & Specificity & PPV & NPV \\
\hline Fukuoka ICG & 62 & 44 & 21 & $66,8 \%$ & $26,8 \%$ & $31,8 \%$ & $61,1 \%$ \\
& & $(71,0 \%)$ & $(33,9 \%)$ & $(14 / 21)$ & $(11 / 41)$ & $(14 / 44)$ & $(11 / 18)$ \\
\hline European EBG & 66 & 61 & 22 & $95,5 \%$ & $11,3 \%$ & $35 \%$ & $83,3 \%$ \\
& & $(92,4 \%)$ & $(33,3 \%)$ & $(21 / 22)$ & $(5 / 44)$ & $(21 / 60)$ & $(5 / 6)$ \\
\hline AGA IG & 52 & 34 & 20 & $80,0 \%$ & $43,8 \%$ & $47,1 \%$ & $77,8 \%$ \\
& & $(65,4 \%)$ & $(38,5 \%)$ & $(16 / 20)$ & $(14 / 32)$ & $(16 / 34)$ & $(14 / 18)$ \\
\hline
\end{tabular}

$\mathrm{N}$ : number of cysts, Criteria + : Fulfilled criteria of the guideline for referral to surgery, PPV : Positive Predictive Value, NPV : Negative Predictive Value.

Table 5. - Performance of the three guidelines

\begin{tabular}{|l|c|c|c|c|c|c|c|c|c|}
\hline & $\mathrm{N}$ & \multicolumn{2}{|c|}{ Avoidable resection } & \multicolumn{2}{c|}{ Missed malignancy } & \multicolumn{2}{|c|}{ Overtreatment } & \multicolumn{2}{c|}{ Correct referral } \\
\hline Fukuoka ICG & Total group & $11 / 62$ & $17,7 \%$ & $7 / 62$ & $11,3 \%$ & $30 / 62$ & $48,4 \%$ & $14 / 62$ & $22,6 \%$ \\
& Asymptomatic & $4 / 29$ & $13,8 \%$ & $2 / 29$ & $6,9 \%$ & $18 / 29$ & $62,0 \%$ & $5 / 29$ & $17,2 \%$ \\
\hline European EBG & Total group & $5 / 66$ & $7,6 \%$ & $1 / 66$ & $1,5 \%$ & $39 / 66$ & $59,1 \%$ & $21 / 66$ & $31,8 \%$ \\
& Asymptomatic & $4 / 32$ & $12,5 \%$ & $1 / 32$ & $3,1 \%$ & $21 / 32$ & $65,6 \%$ & $6 / 32$ & $18,8 \%$ \\
\hline \multirow{2}{*}{ AGA IG } & Total group & $15 / 52$ & $26,9 \%$ & $4 / 52$ & $7,7 \%$ & $18 / 52$ & $34,6 \%$ & $16 / 52$ & $30,8 \%$ \\
& Asymptomatic & $8 / 22$ & $36,4 \%$ & $2 / 22$ & $9,1 \%$ & $9 / 22$ & $40,9 \%$ & $3 / 22$ & $13,6 \%$ \\
\hline
\end{tabular}


For the Fukuoka ICG, there were 62 cysts for which the criteria could be applied in retrospect. $71 \%(44 / 62)$ had positive criteria for referral to surgery, but only $33,9 \%(21 / 62)$ were malignant. This led to a sensitivity of $66,8 \%$, a specificity of $26,8 \%$, a PPV of $31,8 \%$ and a NPV of $61,1 \%$. The accuracy was $40,3 \%$.

For the European EBG guidelines, the criteria were applicable to 66 cysts of which $92,4 \%(61 / 66)$ had positive criteria and $33,3 \%(22 / 66)$ were malignant. This led to a sensitivity of $95,5 \%$, a specificity of $11,3 \%$, a PPV of $35 \%$ and a NPV of $83,3 \%$. The accuracy was $39,4 \%$

Analysing the AGA IG, 52 cysts were included. 65,4\% (34/52) had positive criteria for referral to surgery. The final pathology report confirmed malignancy in $38,5 \%$ (20/52) of the cysts. This led to a sensitivity of $80 \%$, a specificity of $43,8 \%$, a PPV of $47,1 \%$ and a NPV of $77,8 \%$. The accuracy was $57,7 \%$.

The rate of avoidable resections in our cohort group was respectively $17,7 \%, 7,6 \%$ and $26,9 \%$ for the Fukuoka ICG, the European EBG and the AGA IG. If we refined for the group of asymptomatic patients, the rates were $13,8 \%, 12,5 \%$ and $36,4 \%$ respectively. The rates of missed malignancy were $11,3 \%, 1,5 \%$ and $7,7 \%$ respectively or if we refined for the asymptomatic group, missed malignancy rate is $6,9 \%, 3,1 \%$ and $9,1 \%$. The correct referral rate according to the guidelines was respectively $22,6 \%, 31,8 \%$ and $30,8 \%$, but with a lower number in the asymptomatic group. The rates of overtreatment are respectively $48,4 \%, 59,1 \%$ and $34,6 \%$ with a further increase of overtreatment in the asymptomatic subgroup (table 5). However, it would be inaccurate to designate all the non-malignant cysts as overtreatment in our study population. If we took into consideration the group of malignant cysts and the group of cysts which were presumed to be symptomatic with improvement of the symptoms after resection, surgery was justified in $62,5 \%(45 / 72)$.

\section{Discussion}

As the morbidity and mortality of pancreatic surgery is high and the prognosis of pancreatic cancer dismal, a compromise must be found between overtreatment and missed malignancy if pancreatic cysts are considered for surgical resection. The current techniques of MRI and EUS with FNA succeed largely in identifying the correct type of cyst, but the challenge remains to distinguish premalignant cysts - that may be safely followed-up - from high grade dysplasia/malignant cysts. Three guidelines: Fukuoka ICG, European EBG and AGA IG have specifically been developed to identify cysts at risk for invasive carcinoma, but all of them may lead to surgical overtreatment. Our results showed that the European EBG had the best sensitivity of $95,5 \%$ for detecting malignant cysts, followed by the AGA IG with $80 \%$ and the Fukuoka ICG guideline with a sensitivity of $66,8 \%$. The specificity of the three guidelines however was low ranging from $11,3 \%$ for the European EBG up to $26,8 \%$ for the Fukuoka guidelines and $43,8 \%$ for the AGA IG (table 4). With regards to missed malignancy and overtreatment, the European EBG had a low number of missed malignancy $(1,5 \%)$ at the expense of the highest rate of overtreatment $(59,1 \%)$. For the Fukuoka ICG and the AGA IG, the rate of missed malignancy was higher, respectively $11,7 \%$ and $7,7 \%$ with a lower percentage of overtreatment, respectively $48,4 \%$ and $34,6 \%$ (table 5).

Our results for the European EBG were comparable to a previous study (Lekkerkerker et al) in which the authors performed a similar analysis utilizing the 2013 European EBG guideline. They calculated a missed malignancy rate of $0 \%$ (versus $1,5 \%$ in our study) and an avoidable resection rate of $9 \%$ (versus $7,6 \%$ in our study) $(8,9)$. This study, as well as the study by Ma et al showed a missed malignancy rate for the AGA IG of 12 $\%$ (versus $7,7 \%$ in our study) (10). Another retrospective study on 269 resected cysts showed a missed malignancy rate for the AGA IG of $92,6 \%$ which is very high and not comparable to our results (11). The reason for this discrepancy with our data is unclear. For the Fukuoka ICG, as the worrisome features and high risk stigmata are comparable with the European EBG, a similar outcome would be expected between both guidelines as the study of Lekkerkerker et al showed (the missed malignancy rate was for both guidelines in that study $0 \%$ ) (8). However in our study, we saw a remarkable different outcome between the Fukuoka ICG and the European EBG with a missed malignancy rate of $11.3 \%$ versus $1.5 \%$ respectively and a lower sensitivity of $66,8 \%$ versus $95,5 \%$. This discrepancy was partly due to the requirement of confirming worrisome features by EUS in the Fukuoka ICG before referring to surgery which was not necessary for the European EBG (3). If the result of the EUS was inconclusive or there is a cyst size of more than $30 \mathrm{~mm}$ without worrisome features on EUS, the Fukuoka ICG leaves the choice between surveillance or surgery. This section of inconclusive result creates a grey zone in the decision tree of the Fukuoka ICG which makes it difficult to apply the guideline uniformly. In our setting and analysis of the Fukuoka ICG, cysts with inconclusive EUS or cysts with a size above $30 \mathrm{~mm}$ without confirmation of worrisome features by EUS were not an indication for surgery. Secondly for the European EBG, cysts with only one worrisome feature was sufficient for a relative indication for surgery in a patient without significant comorbidities (4). Although relative indication is also not well delineated, the personal interpretation of the clinician and the preference of the patient will be decisive in real life. However, in our retrospective analysis, we designated the cysts with one worrisome feature as a positive advice for referral to surgery in the European EBG. With these differences between the Fukuoka ICG and European EBG in mind, we considered in more detail in our study population the cases of missed malignancy by the Fukuoka ICG which had been correctly identified by the European 
EBG. It concerned six patients of whom four were MD-IPMN and two mixed-type IPMN. These six cysts were also correctly referred for surgery by the AGA IG. The reason why the Fukuoka ICG scored negative for surgery is related to the fact that all six cysts had a dilated MPD between 5 and $9,9 \mathrm{~mm}$ without confirmation of worrisome features by the performed EUS. In general, the risk of $\mathrm{HG}$ dysplasia or invasive carcinoma is estimated $37-91 \%$ for a MPD dilatation of 5 to $9,9 \mathrm{~mm}$ and $56-89 \%$ for a MPD $\geq 10 \mathrm{~mm}(3,4)$. In our study population, 41 patients had a dilated MPD of whom $46 \%$ (19/41) were malignant cysts and 54\% (22/41) were nonmalignant. The mean dilatation of the MPD between the malignant versus the non-malignant group did not differ substantially: 7,69 $\mathrm{mm}$ and 7,78 $\mathrm{mm}$ respectively. On top of that, dilatation of the MPD can also be seen in other pancreatic diseases. Therefore, other aspects of the pancreas like parenchymal calcifications in chronic pancreatitis can contribute to the differential diagnosis. These factors make the definition of a cut off and the interpretation of MPD dilation difficult. Especially if the diameter is between 5 and $9,9 \mathrm{~mm}$, interpretation must be done with caution (12). To illustrate: If we modify the criteria of the Fukuoka ICG by lowering the threshold of MPD dilatation as high risk stigmata from $10 \mathrm{~mm}$ to 7 $\mathrm{mm}$, the performance based on our database would show a sensitivity of $90.5 \%$, specificity of $19.5 \%$ and a missed malignancy rate of $3.2 \%$.

As our and other series have shown, the current practice based on the combination of iconographic features and cytology obtained by FNA has shortcomings resulting in a high number of unnecessary surgery and a minority of missed malignancy. New insights about driver gene alternations (e.g. KRAS, GNAS, etc) have recently been published which may play a role in predicting the risk of progression towards malignancy $(13,14)$. At this moment, there are no implications for daily practice, but this knowledge might change the management of pancreatic cysts in the future.

Although this study gave an idea about the performance of the three guidelines, there are some limitations.

At first, this was a retrospective study which has the risk of selection and information bias.

A second remark is the consistent interpretation of the guidelines. As already mentioned, "the inconclusive result" and "the relative indication for surgery" from the Fukuoka ICG and the European EBG respectively created a grey zone in the applicability of the guidelines. To enable a uniform interpretation in our analysis, we decided arbitrary that the inconclusive result and the relative indication would respectively be a negative and positive advice for referral to surgery. It is possible that other authors or clinicians would disagree with this judgement which would lead to different outcomes. In addition, the quality of EUS is operator dependent and the role of EUS is prominent in our study. However, we believe variation of EUS quality is rather unlikely given the expertise in our referral centre.
A third remark is that $40-50 \%$ of the cysts in our analysis were symptomatic which is a high proportion, probably influencing the decision of referral to surgery and bias our results although we cannot prove this in our retrospective analysis. According to the guidelines (European EBG and Fukuoka ICG) the symptoms that can be attributed to a pancreatic cyst are jaundice and pancreatitis and only the European EBG considers a rapid onset or deterioration of diabetes as a possible symptom. Especially jaundice is a strong argument (the so-called high risk stigmata or absolute indications) for referral to surgery because of the risk of underlying malignancy. the AGA IG is intended only for asymptomatic cysts and does not mention symptoms. In daily practice, a patient with a pancreatic cyst often reports other symptoms such as weight loss, pancreatic like pain or steatorrhea and it is not always clear before the surgery whether or not these symptoms are related to the cyst. In this study, we considered all of the 6 symptoms mentioned in this paragraph (jaundice, pancreatitis, new-onset or deterioration of diabetes, weight loss, steatorrhea and pancreatic like pain) as symptoms that can be related to the pancreatic cyst. As such, 36 symptomatic cysts were identified, however in 7 of these 36 cysts it was rather unclear to argue in retrospect whether the treating physician considered at the time the reported symptoms to be related or not.

A fourth remark is the classification of mucinous cysts with low grade dysplasia as premalignant cysts. Once the cyst is resected, the natural evolution of the cyst is lost and the risk of malignancy if not resected cannot be assessed which attenuates the connotation of overtreatment by surgery of premalignant cysts.

A fifth remark is that our centre is a third line referral centre for pancreatic surgery. This can lead to a selection bias.

At last, we did not subdivide $\mathrm{HG}$ dysplasia and invasive carcinoma in our analysis of the performance of the guidelines. These two pathological outcomes are gathered in one group as "malignant cysts". As expected, the prognosis of resected $\mathrm{HG}$ dysplasia was better than for resected invasive adenocarcinoma: The 3-year survival rate for resected HG Dysplasia was 90\% (9/10 - the death of that one patient was not cancer or surgery related), the 3 -year survival rate for resected adenocarcinoma was $27 \%$ (3/11 - all cancer related deaths).

\section{Conclusion}

The aim in the management of pancreatic cysts is finding a compromise between preventing unnecessary surgery and avoiding missed malignancy. This retrospective analysis showed that the European EBG had the least missed malignancy at the expense of a higher rate of overtreatment. The Fukuoka ICG had the highest amount of missed malignancy. This high number of false negative cases could partially be explained by the 
strict requirement of EUS confirmation for worrisome features before referral to surgery. The AGA IG had the lowest rate of overtreatment, but also a high rate of missed malignancy. Our analysis also demonstrated that dilatation of the MPD between 5 and $9,9 \mathrm{~mm}$ is a grey zone for malignancy and must be interpreted with caution. There is a need to develop better biomarkers to predict the risk of malignancy of pancreatic cysts.

\section{Conflicts of interest}

None of the authors have any conflicts of interest.

\section{References}

1. STARK A., DONAHUE T.R., REBER H.A., HINES O.J. Pancreatic cyst disease: a review. JAMA, 2016, $315:$ 1882-1893.

2. SCHOLTEN L., VAN HUIJGEVOORT N.C.M., VAN HOOFT J.E., BESSELINK M.G., DEL CHIARO M. Pancreatic cystic neoplasms : different types, different management, new guidelines. Visc. Med., 2018, 34 : 173-177.

3. TANAKA M., FERNANDEZ-DEL CASTILLO C., KAMISAWA T., JANG J.Y., LEVY P., OHTSUKA T. et al. Revisions of international consensus Fukuoka guidelines for the management of IPMN of the pancreas. Pancreatology, 2017, 17 : 738-753.

4. EUROPEAN STUDY GROUP ON CYSTIC TUMOURS OF THE PANCREAS. European evidence-based guidelines on pancreatic cystic neoplasms. Gut, 2018, 67 : 789-804.

5. VEGE S.S., ZIRING B., JAIN R., MOAYYEDI P., CLINICAL GUIDELINES COMMITTEE. American gastroenterological association institute guideline on the diagnosis and management of asymptomatic neoplastic pancreatic cysts. Gastroenterology, 2015, $148: 819-822$.

6. FARRELL J.J. Pancreatic cysts and guidelines. Dig. Dis. Sci., 2017, 62 : 1827-1839.

7. BARKIN J.A., BARKIN J.S. Pancreatic cysts: controversies, advances, diagnoses, and therapies. Pancreas, 2017, $46:$ 735-741.

8. LEKKERKERKER S.J., BESSELINK M.G., BUSCH O.R., VERHEIJ J., ENGELBRECHT M.R., RAUWS E.A. et al. Comparing 3 guidelines on the management of surgically removed pancreatic cysts with regard to pathological outcome. Gastrointest. Endosc., 2017, 85 : 1025-1031.

9. DEL CHIARO M., VERBEKE C., SALVIA R., KLÖPPEL G., WERNER J., MCKAY C. et al. European experts consensus statement on cystic tumours of the pancreas. Dig. Liver Dis., 2013, $45:$ 703-711.

10. MA G.K., GOLDBERG D.S., THIRUVENGADAM N., CHANDRASEKHARA V., KOCHMAN M.L., GINSBERG G.G. et al. Comparing American Gastroenterological Association pancreatic cyst management guidelines with Fukuoka consensus guidelines as predictors of advanced neoplasia in patients with suspected pancreatic cystic neoplasms. J. Am. Coll. Surg., 2016, 223 : 729-737.

11. XU M.M., YIN S., SIDDIQUI A.A., SALEM R.R., SCHROPE B., SETHI A. et al. Comparison of the diagnostic accuracy of three current guidelines for the evaluation of asymptomatic pancreatic cystic neoplasms. Medicine, 2017, 96 : e7900.

12. MARCHEGIANI G., ANDRIANELLO S., MORBIN G., SECCHETTIN E., D'ONOFRIO M., DE ROBERTIS R. et al. Importance of main pancreatic duct dilatation in IPMN undergoing surveillance. Br. J. Surg., 2018, 105 : 1825-1834.

13. OMORI Y., ONO Y., TANINO M., KARASAKI H., YAMAGUCHI H., FURUKAWA T. et al. Pathways of progression from intraductal papillary mucinous neoplasm to pancreatic ductal adenocarcinoma based on molecular features. Gastroenterology, 2019, $156:$ 647-661.

14. SPRINGER S., WANG Y., DAL MOLIN M., MASICA D.L., JIAO Y. KINDE I. et al. A combination of molecular markers and clinical features improve the classification of pancreatic cysts. Gastroenterology, 2015, 149 1501-1510. 